\title{
Factors associated with Health-Related Quality of Life in Hispanic population with mental disorders using medical expenditure panel survey 2013-2017
}

\author{
Jongwha Chang*1, Jangkwon $\mathrm{Cho}^{2}$, Mar Medina ${ }^{2}$, Stephanie Falcon ${ }^{2}$, Paulina Soto-Ruiz ${ }^{2}$, Dong Yeong Shin ${ }^{3}$ \\ ${ }^{1}$ Department of Healthcare Administration, Texas Women's University, College of Business, Denton, Texas, United States \\ ${ }^{2}$ Department of Pharmaceutical Sciences, University of Texas, School of Pharmacy, El Paso, Texas, United States \\ ${ }^{3}$ Department of Public Health Sciences, New Mexico State University, Las Cruces, New Mexico, United States
}

Received: April 5, 2021

DOI: $10.5430 /$ jha.v10n3p1
Accepted: April 22, 2021

Online Published: April 26, 2021

\begin{abstract}
There is a lack of U.S. population-based research surrounding the marked decrease in health-related quality of life (HRQoL) caused by the morbidity of mental disorders in the U.S. Hispanic demographic. This cross-sectional study utilized data from the 2013-2017 Medical Expenditure Panel Survey (MEPS) to identify Hispanic community-dwelling residents with mental disorders in the U.S. The independent variable was the presence of mental disorders, and the dependent variable was HRQoL. HRQoL was measured with the Short Form 12 (SF-12) Physical Health Composite Scale (PCS) and Mental Health Composite Scale (MCS). A total of 34,434 patients met the inclusion criteria, representing about 38,683,299 Hispanic individuals. Of this group, those older than 18 were stratified by the presence of mental disorders. The two groups were those with mental disorders: 4,122 individuals representing a sample size of 4,789,634; and those without mental disorders: 30,312 individuals representing a sample size of $33,893,665$. Based on our study, Hispanic patients with mental disorders were associated with lower HRQoL scores. SF-12 PCS scores $(95 \% \mathrm{CI})$ were $45.3(44.5,46.1)$ for those with mental disorders and $50.8(50.5,51.0)$ for those without mental disorders. SF-12 MCS scores $(95 \% \mathrm{CI})$ were $42.6(42,43.3)$ in patients with mental disorders and $52.6(52.3,52.8)$ in patients without mental disorders. These differences in scores denote the impact of mental health disorders on HRQoL scores in the Hispanic demographic and mark the way for further research on identifying means of improving such scores for Hispanic patients.
\end{abstract}

Key Words: Menta Disorders, Health-related quality of life, Hispanic Population, Medical Expenditure Panel Survey

\section{INTRODUCTION}

Mental health disorder is an illness that affects an individual's work, school, relationships, and ability to adapt and cope with adversity. ${ }^{[1]}$ Types of mental disorders include anxiety, panic, obsessive-compulsive disorder, phobia, depression, bipolar, eating disorder. ${ }^{[2]}$ According to the American Psychiatric Association, nearly 1 out 5 U.S adults experience some kind of mental illness, and 1 in 24 develop a severe mental illness. ${ }^{[1-3]}$ The National Library of Medicine states that more than half of all Americans will be diagnosed with a mental disorder at some time in their life. ${ }^{[2]}$ Factors that may lead to the development of a mental disorder include family history, traumatic life experience, brain injury, chemical imbalances, and the use of alcohol and recreational drugs. ${ }^{[2]}$ Fortunately, treatment is available for mental health disor-

\footnotetext{
* Correspondence: Jongwha Chang; Email: jochang@umich.edu; Address: Department of Healthcare Administration, College of Business, Texas Women's University, 304 Administration Dr. Denton, TX 76204, United States.
} 
ders. ${ }^{[1]}$ However, disparities in access and quality of care exist amongst different populations and cultures. ${ }^{[4,5]}$

The Hispanic population is one of the fastest-growing racial groups in the United States. ${ }^{[4]}$ At about 60.4 million, the Hispanic population makes up $18.3 \%$ of the U.S. population. ${ }^{[4,6]}$ By 2060, the Hispanic population is projected to grow to 119 million, which will be about $28.6 \%$ of the U.S. population. ${ }^{[4,6]}$ Currently, $16 \%$ of the Hispanic population in the U.S. is reported to have mental health disorders, and only about $33 \%$ receive treatment. ${ }^{[3,7]}$ Previous studies have reported that more significant mental health care disparities exist for the Hispanic population than for non-Hispanic whites. ${ }^{[5]}$ Some barriers contributing to this disparity are language barriers, socioeconomic barriers, education, health insurance coverage, cultural competence, and legal status. ${ }^{[7]}$ In addition to morbidity and mortality, Health-Related Quality of Life (HRQoL) is becoming a vital patient factor in determining many diseases' treatment outcomes. ${ }^{\left[{ }^{[, 9]}\right.}$ HealthRelated Quality of Life is a subjective and multidimensional concept that assesses an individual's physical, mental, and social function and well-being. ${ }^{[10,11]}$ This concept helps quantify how the patient's physical or mental conditions limit their daily living, social experiences, and mental wellbeing. ${ }^{[10,11]}$ The inclusion of HRQoL data has proven to increase the accuracy of survival prognostication. ${ }^{[8]}$ Patients with higher HRQoL have been found to have lower mortality risk compared to those with the same health condition and lower HRQoL. ${ }^{[8]}$ Multiple studies have shown that numerous factors can impact an individual's HRQoL. ${ }^{[12-14]}$ These factors include age, gender, race, ethnicity, obesity, socioeconomic status, socio-ecological factors, and health system factors. ${ }^{[10,12-14]}$ Furthermore, individuals with mental health conditions tend to experience lower HRQoL than individuals with other chronic medical conditions. ${ }^{[5]}$ Mental health disorders correlate with higher prevalence of heavy drinking, smoking, physical inactivity, and obesity- all of which are factors that affect an individual's HRQoL. ${ }^{[15]} \mathrm{HRQoL}$ disparities also exist between different races and ethnicities. ${ }^{[14]}$ The Hispanic population experiences lower HRQoL due to lower socioeconomic status caused by differences in immigration status, education, employment, financial status, and health care access. ${ }^{[16,17]}$ Health-Related Quality of Life data gathered from the Behavior Risk Factor Surveillance System showed that twice as many Hispanics reported fair or poor health conditions than non-Hispanic Whites. ${ }^{[10]}$ Hispanics were also more likely to report more frequent mental distress than non-Hispanic Whites. ${ }^{[10]}$

This study aims to identify what HRQoL factors most affect the HRQoL of mental health patients in the Hispanic population. The study will help determine what HRQoL disparities exist between Hispanic mental health patients and Hispanic non-mental health patients, prioritizing changes required to help improve the HRQoL of mental health patients in the Hispanic population.

\section{Methods}

\subsection{Data source}

Data from the 2013-2017 Medical Expenditure Panel Survey (MEPS) were used to conduct a retrospective data study. MEPS provides a complete source of data on the cost and use of healthcare and health insurance coverage through large-scale surveys on families, individuals, and healthcare employees in the United States. MEPS focuses on healthcare access, coverage, and cost using self and caregiver-reported information. Each year, MEPS provides continuous and current estimates of healthcare utilization and expenditure at the individual and household levels. Because MEPS data is publicly available de-identified data, Institutional Review Board (IRB) approval was waived.

\subsection{Patient selection and outcome measures}

From the MEPS database, Hispanic patients who had positive weight values and were older than 18 were included in the analyses. The Hispanic and Non-Hispanic population was categorized based on the Hispanic ethnicity variables (HISPANX). Patients with a diagnosis of a mental disorder were identified using International Classification of Diseases, Tenth Revision, Clinical Modification (ICD-10-CM: 295, 296, 298, 300, 309, 311, F20, F31, F32, F33, F40, F41, F42, F99) and Clinical Classification Code (CCC: 651, 650, 657, 659). The code list of mental disorders was created based on the category determined by AHRQ.[18] Selected patients were older than 18 and had at least one SF-12 PCS or MCS score.

PCS and MCS are computed using the scores of twelve questions. The questions have three or five-level Likert scales that measure health levels; a lower score indicates a low level of health while a higher score indicates a high level of health. ${ }^{[19]}$ The PCS and MCS scoring algorithms incorporate information from all the questions. More heavily weighted questions were addressed for PCS and MCS. In PCS, the heavily weighted questions were questions $1,2,3,4,5$, and 8 , and for MCS, these questions were questions 6,7,9,11, and $12 .^{[20]}$

Andersen's Behavioral Model was used to assess factors associated with QOL in patients with mental disorders. ${ }^{[21]}$ Five predictors from the conceptual framework developed the independent variables. These predictors were: predisposingrace, Hispanic ethnicity, and age; enabling- marital status, education, employment, poverty status, insurance coverage, 
and usual source of care; need- perceived physical and mental health status, body mass index (BMI), and other chronic conditions; personal health practices- smoking status; and external environment- geographical region.

Factor variables were created for each mutually exclusive demographic category. Age in years was defined as those under 18, 18-44, and 56-64. Race was separated into Hispanic-white and other/multiple races. Educational status categories were no degree or less than high school, high school, and some college. Four geographical regions were defined as Northeast, Midwest, South, and West. Marital status was divided into married and unmarried (widowed, divorced/separated, or never married). Smoking status and employment were also determined as yes or no. Type of health insurance was categorized as public, private, or uninsured. Poverty level was defined as poor/low income (less than $200 \%$ ), middle income (200\% to less than $400 \%$ ), and high income (greater than or equal to $400 \%$ ). Perceived health status and perceived mental health status were both categorized as fair/poor, good, or excellent/very good. BMI was separated into underweight/normal less than 24.9, overweight 25.0-29.9, and obese more than 30.0.

\subsection{Statistical analysis}

Since MEPS uses a complex multistage sampling design to represent the overall population, individual responses must be weighted by the proportion of their representative population. Individual weights in MEPS were used to derive national estimates of demographics and socioeconomic covariates. The number of respondents with mental disease and the weighted sample size were presented. Unique weighted variables designed for the self-administration questionnaire (SAQ) for people 18 and older were used to weigh the SF-12 scores. The weighted variables adjust for SAQ non-respondents and accounts for the U.S. civilian noninstitutionalized population. ${ }^{[20]}$ Multivariate regression models controlling for covariates were performed to predict MCS and PCS scores amongst the Hispanic population. The "proc survey" procedure of SAS and the "svy" procedure of Stata calculated accurate point estimates and their standard errors for the nationally representative population with MEPS survey weights. ${ }^{[22]}$ All analyses were conducted using SAS version 9.4 (SAS Institute Inc., Cary, NC) and Stata version 12 (Stata Corp. LP, College Station, TX). All statistical analyses were 2-tailed, and the significance level was set a priori at $p<.05$.

\section{Results}

A total of 166,098 respondents with positive individual weights were identified, representing a weighted sample size of $320,701,450$. Hispanic respondents older than 18 were stratified by the existence of mental disorder: Mental disorder: 4,122 (weighted sample size: 4,789,634) and NonMental disorder: 30,312 (weighted sample size: 33,893,665). Most patients were of Mexican or Mexican American descent at $46.3 \%$, followed by those of Cuban or Dominican descent at $23.4 \%$. The mean age was 56.76 years with a standard deviation $(S D)$ of 1.52 years (see Figure 1).

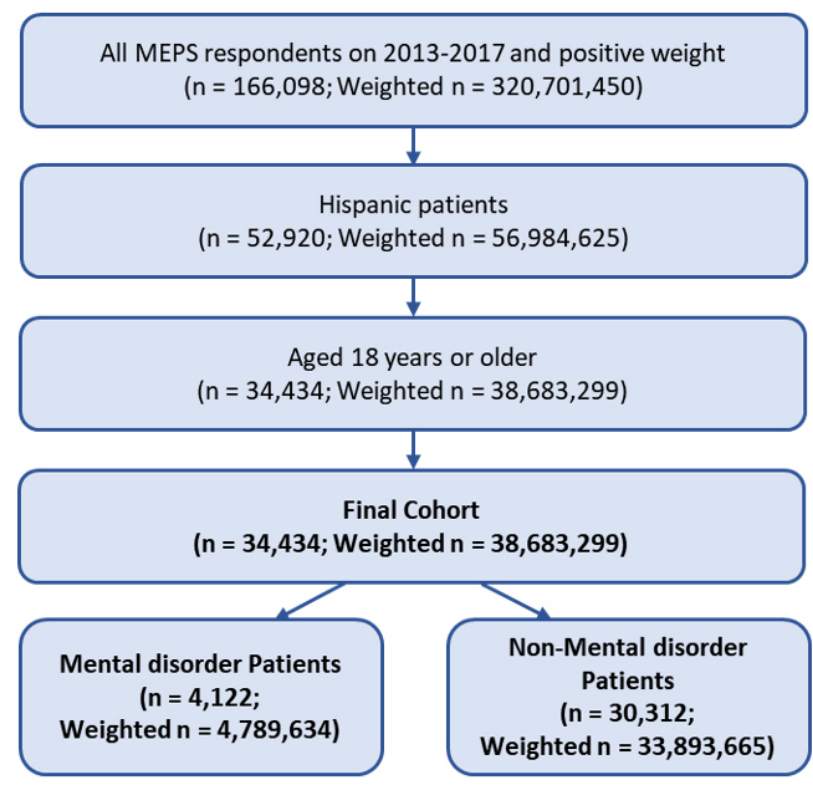

Figure 1. Patient attrition

Hispanic white accounted for $93.3 \%$ of all respondents. For overall groups, $46.8 \%$ had private insurance, and $40.45 \%$ had public insurance only. The mental disorder group had a higher percentage of those with poor or low income (48.8\%) compared to the non-mental disorder group $(42.1 \%)$ ( $p<$ $.001)$. The mental disorder group had a higher percentage of diabetes, hypertension, high cholesterol, heart disease, arthritis, and cancer compared to the non-mental disorder group $(p<.001)$ (see Table 1$)$.

Tables 2 and 3 represent the unadjusted SF-12 scores for PCS and MCS amongst Hispanic patients with mental disorders. The average of SF-12 PCS was 45.3 (95\% CI: 44.5-46.1). Patients aged 18-44 had a PCS score of 50.3 (95\% CI: 49.551.0), 13.3 points higher than patients older than 65 who scored 37.0 (95\% CI: 35.4-38.5). Patients who perceived excellent or very good health status had a PCS score of 46.8 (95\% CI: 45.7-47.9), 10.2 points higher than those with fair or poor health status 36.6 (95\% CI: 35.6-37.6). Similarly, well-educated patients had a score of 47.9 (95\% CI: 47.048.8), 6 points higher than less-educated patients at a score of 41.9 (95\% CI: 40.6-43.2). 
Table 1. Demographics and clinics characteristics of Hispanic patients with/without mental disorder

\begin{tabular}{|c|c|c|c|c|c|c|c|c|c|c|}
\hline & \multicolumn{3}{|c|}{ Overall } & \multicolumn{3}{|c|}{ Mental disorder } & \multicolumn{3}{|c|}{ Non-Mental disorder } & \multirow[b]{2}{*}{$p$-value } \\
\hline & $\mathbf{n}$ & $\begin{array}{c}\text { Weighted } \\
\text { N }\end{array}$ & $\begin{array}{c}\text { Weighted } \\
\%\end{array}$ & $\mathbf{n}$ & $\begin{array}{l}\text { Weighte } \\
\text { d N }\end{array}$ & $\begin{array}{c}\text { Weighted } \\
\%\end{array}$ & $\mathbf{n}$ & $\begin{array}{l}\text { Weighted } \\
\text { N }\end{array}$ & $\begin{array}{c}\text { Weighted } \\
\%\end{array}$ & \\
\hline Total & 34,434 & $38,683,299$ & $100.0 \%$ & 4,122 & $4,789,634$ & $100.0 \%$ & 30,312 & $33,893,665$ & $100.0 \%$ & \\
\hline \multicolumn{11}{|l|}{ Age } \\
\hline Mean $(S D)$ & $41.39(0.2)$ & & & $45.37(0.48)$ & & & $40.82(0.2)$ & & & .000 \\
\hline $18-44$ & 20,513 & $23,645,301$ & $61.1 \%$ & 1,917 & $2,459,781$ & $51.4 \%$ & 18,596 & $21,185,520$ & $62.5 \%$ & .000 \\
\hline $45-64$ & 10,449 & $11,037,394$ & $28.5 \%$ & 1,543 & $1,585,937$ & $33.1 \%$ & 8,906 & $9,451,457$ & $27.9 \%$ & \\
\hline$\geq 65$ & 3,472 & $4,000,604$ & $10.3 \%$ & 662 & 743,915 & $15.5 \%$ & 2,810 & $3,256,688$ & $9.6 \%$ & \\
\hline \multicolumn{11}{|l|}{ Race/ethnicity } \\
\hline White & 32,321 & $36,077,933$ & $93.3 \%$ & 3,734 & $4,319,997$ & $90.2 \%$ & 28,587 & $31,757,936$ & $93.7 \%$ & .001 \\
\hline Black & 764 & 922,510 & $2.4 \%$ & 123 & 155,537 & $3.2 \%$ & 641 & 766,972 & $2.3 \%$ & \\
\hline Others & 1,349 & $1,682,856$ & $4.4 \%$ & 265 & 314,099 & $6.6 \%$ & 1,084 & $1,368,757$ & $4.0 \%$ & \\
\hline \multicolumn{11}{|l|}{ Region } \\
\hline Northeast & 5,015 & $5,345,668$ & $13.8 \%$ & 840 & 846,465 & $17.7 \%$ & 4,175 & $4,499,203$ & $13.3 \%$ & .000 \\
\hline Midwest & 3,019 & $3,429,659$ & $8.9 \%$ & 432 & 567,051 & $11.8 \%$ & 2,587 & $2,862,609$ & $8.4 \%$ & \\
\hline South & 12,215 & $14,475,561$ & $37.4 \%$ & 1,180 & $1,417,844$ & $29.6 \%$ & 11,035 & $13,057,717$ & $38.5 \%$ & \\
\hline West & 14,185 & $15,432,411$ & $39.9 \%$ & 1,670 & $1,958,274$ & $40.9 \%$ & 12,515 & $13,474,137$ & $39.8 \%$ & \\
\hline \multicolumn{11}{|l|}{ Insurance Type } \\
\hline Any private & 13,736 & $19,051,606$ & $49.3 \%$ & 1,514 & $2,240,500$ & $46.8 \%$ & 12,222 & $16,811,106$ & $49.6 \%$ & .000 \\
\hline Public only & 10,290 & $9,532,215$ & $24.6 \%$ & 1,976 & $1,934,604$ & $40.4 \%$ & 8,314 & $7,597,611$ & $22.4 \%$ & \\
\hline Uninsured & 10,408 & $10,099,478$ & $26.1 \%$ & 632 & 614,530 & $12.8 \%$ & 9,776 & $9,484,949$ & $28.0 \%$ & \\
\hline \multicolumn{11}{|l|}{ Marital Status } \\
\hline Married & 15,802 & $18,159,043$ & $46.9 \%$ & 1,560 & $1,897,929$ & $39.6 \%$ & 14,242 & $16,261,114$ & $48.0 \%$ & .000 \\
\hline Unmarried & 18,632 & $20,524,256$ & $53.1 \%$ & 2,562 & $2,891,705$ & $60.4 \%$ & 16,070 & $17,632,551$ & $52.0 \%$ & \\
\hline \multicolumn{11}{|c|}{ Education Attainment } \\
\hline $\begin{array}{l}\text { Less than high } \\
\text { school }\end{array}$ & 13,674 & $12,482,526$ & $32.3 \%$ & 1,577 & $1,408,720$ & $29.4 \%$ & 12,097 & $11,073,806$ & $32.7 \%$ & .000 \\
\hline High school & 9,640 & $11,022,960$ & $28.5 \%$ & 1,039 & $1,154,950$ & $24.1 \%$ & 8,601 & $9,868,010$ & $29.1 \%$ & \\
\hline College or higher & 11,120 & $15,177,813$ & $39.2 \%$ & 1,506 & $2,225,964$ & $46.5 \%$ & 9,614 & $12,951,848$ & $38.2 \%$ & \\
\hline \multicolumn{11}{|l|}{ Family Income ${ }^{\#}$} \\
\hline Negative/poor/low & 18,495 & $16,614,909$ & $43.0 \%$ & 2,464 & $2,339,484$ & $48.8 \%$ & 16,031 & $14,275,425$ & $42.1 \%$ & .000 \\
\hline Middle & 10,508 & $12,750,813$ & $33.0 \%$ & 1,077 & $1,386,650$ & $29.0 \%$ & 9,431 & $11,364,163$ & $33.5 \%$ & \\
\hline High & 5,431 & $9,317,577$ & $24.1 \%$ & 581 & $1,063,500$ & $22.2 \%$ & 4,850 & $8,254,077$ & $24.4 \%$ & \\
\hline \multicolumn{11}{|l|}{ Current Smoking } \\
\hline Yes & 7,653 & $8,582,972$ & $22.2 \%$ & 1,044 & $1,145,856$ & $23.9 \%$ & 6,609 & $7,437,116$ & $21.9 \%$ & .048 \\
\hline No & 26,781 & $30,100,327$ & $77.8 \%$ & 3,078 & $3,643,778$ & $76.1 \%$ & 23,703 & $26,456,549$ & $78.1 \%$ & \\
\hline Health Status & & & & & & & & & & \\
\hline $\begin{array}{l}\text { Excellent/Very } \\
\text { good }\end{array}$ & 16,807 & $20,659,046$ & $53.4 \%$ & 1,134 & $1,585,296$ & $33.1 \%$ & 15,673 & $19,073,750$ & $56.3 \%$ & .000 \\
\hline Good & 11,506 & $12,092,515$ & $31.3 \%$ & 1,349 & $1,574,129$ & $32.9 \%$ & 10,157 & $10,518,387$ & $31.0 \%$ & \\
\hline Fair/poor & 6,121 & $5,931,738$ & $15.3 \%$ & 1,639 & $1,630,209$ & $34.0 \%$ & 4,482 & $4,301,529$ & $12.7 \%$ & \\
\hline Diabetes & & & & & & & & & & \\
\hline Yes & 3,786 & $3,941,341$ & $10.2 \%$ & 875 & 904,965 & $18.9 \%$ & 2,911 & $3,036,376$ & $9.0 \%$ & .000 \\
\hline No & 30,648 & $34,741,959$ & $89.8 \%$ & 3,247 & $3,884,669$ & $81.1 \%$ & 27,401 & $30,857,290$ & $91.0 \%$ & \\
\hline Hypertension & & & & & & & & & & \\
\hline Yes & 8,610 & $9,347,037$ & $24.2 \%$ & 1,817 & $1,962,736$ & $41.0 \%$ & 6,793 & $7,384,301$ & $21.8 \%$ & .000 \\
\hline No & 25,824 & $29,336,263$ & $75.8 \%$ & 2,305 & $2,826,898$ & $59.0 \%$ & 23,519 & $26,509,364$ & $78.2 \%$ & \\
\hline High cholesterol & & & & & & & & & & \\
\hline Yes & 8,641 & $9,485,716$ & $24.5 \%$ & 1,690 & $1,824,483$ & $38.1 \%$ & 6,951 & $7,661,234$ & $22.6 \%$ & .000 \\
\hline No & 25,793 & $29,197,583$ & $75.5 \%$ & 2,432 & $2,965,151$ & $61.9 \%$ & 23,361 & $26,232,432$ & $77.4 \%$ & \\
\hline Heart disease & & & & & & & & & & \\
\hline Yes & 3,173 & $3,546,103$ & $9.2 \%$ & 886 & 982,677 & $20.5 \%$ & 2,287 & $2,563,426$ & $7.6 \%$ & .000 \\
\hline No & 31,261 & $35,137,196$ & $90.8 \%$ & 3,236 & $3,806,957$ & $79.5 \%$ & 28,025 & $31,330,240$ & $92.4 \%$ & \\
\hline Arthritis & & & & & & & & & & \\
\hline Yes & 5,208 & $5,673,592$ & $14.7 \%$ & 1,459 & $1,526,506$ & $31.9 \%$ & 3,749 & $4,147,086$ & $12.2 \%$ & .000 \\
\hline No & 29,226 & $33,009,707$ & $85.3 \%$ & 2,663 & $3,263,128$ & $68.1 \%$ & 26,563 & $29,746,579$ & $87.8 \%$ & \\
\hline Cancer & & & & & & & & & & \\
\hline Yes & 1,256 & $1,493,694$ & $3.9 \%$ & 296 & 356,626 & $7.4 \%$ & 960 & $1,137,068$ & $3.4 \%$ & .000 \\
\hline No & 33,178 & $37,189,605$ & $96.1 \%$ & 3,826 & $4,433,008$ & $92.6 \%$ & 29,352 & $32,756,598$ & $96.6 \%$ & \\
\hline Year & & & & & & & & & & \\
\hline 2013 & 7,472 & $7,330,196$ & $18.9 \%$ & 932 & 916,944 & $19.1 \%$ & 6,540 & $6,413,252$ & $18.9 \%$ & .238 \\
\hline 2014 & 6,879 & $7,536,445$ & $19.5 \%$ & 832 & 977,031 & $20.4 \%$ & 6,047 & $6,559,414$ & $19.4 \%$ & \\
\hline 2015 & 7,147 & $7,748,234$ & $20.0 \%$ & 900 & $1,027,695$ & $21.5 \%$ & 6,247 & $6,720,539$ & $19.8 \%$ & \\
\hline 2016 & 7,113 & $7,946,669$ & $20.5 \%$ & 809 & 959,184 & $20.0 \%$ & 6,304 & $6,987,485$ & $20.6 \%$ & \\
\hline 2017 & 5,823 & $8,121,755$ & $21.0 \%$ & 649 & 908,779 & $19.0 \%$ & 5,174 & $7,212,976$ & $21.3 \%$ & \\
\hline
\end{tabular}

Note. ${ }^{~ L o w ~}=$ family income $<200 \%$ of poverty line; middle = family income $200 \%-400 \%$ of poverty line; high $=$ family income $>400 \%$ of poverty line. $P$-values were obtained from Chi-2 by comparing the prevalence of depression between cancer survivors and non-cancer survivors. A matched sample will be selected.

In total, the SF-12 MCS score for Hispanic patients with is 6.1 points lower than those with private insurance [45.5 mental disorders was 42.6 (95\% CI: 42.0-43.3). In terms of (95\% CI: 44.5-46.4)] and 3 points lower than those with census regions, patients in the Midwest had the highest MCS public insurance (42.4 (95\% CI: 40.9-43.8)). Those who scores at 43.3 (95\% CI: 41.1-45.6). Patients without insur- perceived their health status as fair or poor had MCS scores ance had MCS scores of 39.4 (95\% CI: 38.5-40.4) which of 38.4 (95\% CI: 37.3-39.4), significantly less than those 
who perceived their health status as excellent or very good [46.2 (95\% CI: 45.1-47.3)].

Factors associated with HRQoL in the Hispanic population with mental disorders are summarized in Table 4 using the multiple regression analysis. In the multiple regression analysis of the SF-12 PCS scores, age, census region, poverty level, perceived health status, BMI, and employment were associated with SF-12 scores. Controlling for covariates, the PCS scores in patients aged more than 65 were significantly lower than the $18-44$ age group by $4.16(\beta=-4.16, p<.001)$. Poverty level impacted PCS, as reflected by the significantly higher score from high-income patients compared to lowincome patients $(\beta=0.97, p<.001)$. Perceived health status was positively associated with PCS scores. Patients with fair or poor health had scored -7.71 lower than those with excellent or very good health $(\beta=-7.71, p<.001)$. Similarly, non-smoking patients had higher scores than smoking patients $(\beta=3.22, p<.001)$.

The multiple regression analysis conducted on the SF-12 MCS scores emphasizes that geographic region, insurance type, marital status, perceived health status, education attainment, and smoking status are associated with SF-12 MCS scores. Patients in the Midwest and South had significantly lower scores than those in the West $(p<.05)$. Type of insurance significantly affected SF-12 MCS scores. No insurance $(\beta=-0.51, p=.057)$ and public insurance $(\beta=-1.14, p<$ $.001)$ had lower MCS scores than those with private insurance. Patients with fewer comorbidities had higher MCS scores $(\beta=4.11, \mathrm{SE}=1.85, p=.027)$. Perceived health status was positively associated with MCS scores. Patients with fair or poor health had scored -5.68 lower than those with excellent or very good health $(\beta=-5.68, p<.001)$. Similarly, non-smoking patients had higher scores than smoking patients $(\beta=4.30, p<.001)$.

Table 2. Physical Component Summary (PCS)

\begin{tabular}{|c|c|c|c|c|c|c|c|c|c|c|}
\hline \multirow[b]{2}{*}{ Category } & & \multicolumn{3}{|c|}{ Overall } & \multicolumn{3}{|c|}{ Mental Disorder } & \multicolumn{3}{|c|}{ Non-Mental Disorder } \\
\hline & & Mean & $\begin{array}{c}\text { Lower } \\
\text { 95\% CI }\end{array}$ & $\begin{array}{c}\text { Upper } \\
95 \% \text { CI }\end{array}$ & Mean & $\begin{array}{c}\text { Lower } \\
95 \% \text { CI }\end{array}$ & $\begin{array}{c}\text { Upper } \\
95 \% \text { CI }\end{array}$ & Mean & $\begin{array}{c}\text { Lower } \\
95 \% \text { CI }\end{array}$ & $\begin{array}{c}\text { Upper } \\
95 \% \text { CI }\end{array}$ \\
\hline Overall & & 50.0 & 49.8 & 50.3 & 45.3 & 44.5 & 46.1 & 50.8 & 50.5 & 51.0 \\
\hline \multirow{3}{*}{ Age } & $18-44$ & 52.8 & 52.5 & 53.0 & 50.3 & 49.5 & 51.0 & 53.1 & 52.8 & 53.3 \\
\hline & $45-64$ & 47.5 & 47.0 & 48.0 & 41.5 & 40.3 & 42.8 & 48.6 & 48.1 & 49.1 \\
\hline & $\geq 65$ & 40.8 & 39.9 & 41.8 & 37.0 & 35.4 & 38.5 & 41.8 & 40.7 & 42.8 \\
\hline \multirow[b]{2}{*}{ Race } & White & 50.0 & 49.7 & 50.3 & 45.0 & 44.2 & 45.9 & 50.7 & 50.5 & 51.0 \\
\hline & Black & 50.7 & 49.5 & 52.0 & 49.4 & 46.3 & 52.4 & 51.0 & 49.7 & 52.4 \\
\hline \multirow{4}{*}{ Region } & Northeast & 49.2 & 48.4 & 50.0 & 43.2 & 41.3 & 45.1 & 50.4 & 49.7 & 51.1 \\
\hline & Midwest & 50.3 & 49.1 & 51.4 & 44.5 & 41.5 & 47.4 & 51.5 & 50.9 & 52.2 \\
\hline & South & 50.2 & 49.7 & 50.6 & 44.8 & 43.3 & 46.3 & 50.8 & 50.4 & 51.3 \\
\hline & West & 50.2 & 49.8 & 50.5 & 46.8 & 45.8 & 47.8 & 50.7 & 50.3 & 51.1 \\
\hline \multirow{3}{*}{$\begin{array}{l}\text { Insurance } \\
\text { Type }\end{array}$} & Any private & 51.6 & 51.3 & 51.9 & 49.0 & 48.1 & 49.9 & 52.0 & 51.7 & 52.3 \\
\hline & Public only & 45.2 & 44.6 & 45.9 & 40.0 & 38.8 & 41.2 & 46.6 & 46.0 & 47.3 \\
\hline & Uninsured & 51.6 & 51.2 & 52.0 & 48.3 & 46.7 & 49.8 & 51.8 & 51.4 & 52.3 \\
\hline Marital & Married & 49.7 & 49.3 & 50.0 & 44.7 & 43.6 & 45.8 & 50.3 & 49.9 & 50.6 \\
\hline Status & Unmarried & 50.4 & 50.1 & 50.7 & 45.7 & 44.7 & 46.7 & 51.2 & 50.9 & 51.5 \\
\hline \multirow{2}{*}{$\begin{array}{l}\text { Education } \\
\text { Attainment }\end{array}$} & High school & 50.4 & 50.0 & 50.7 & 44.8 & 43.4 & 46.3 & 51.1 & 50.7 & 51.4 \\
\hline & College or higher & 51.5 & 51.1 & 51.8 & 47.9 & 47.0 & 48.8 & 52.1 & 51.7 & 52.5 \\
\hline \multirow{3}{*}{$\begin{array}{l}\text { Family } \\
\text { Income }\end{array}$} & Negative/poor/low & 50.7 & 50.3 & 51.1 & 47.2 & 46.1 & 48.3 & 51.2 & 50.8 & 51.6 \\
\hline & Middle & 51.9 & 51.4 & 52.4 & 49.9 & 48.5 & 51.2 & 52.2 & 51.7 & 52.7 \\
\hline & High & 48.6 & 48.2 & 49.0 & 42.3 & 41.2 & 43.4 & 49.7 & 49.3 & 50.1 \\
\hline Current & Yes & 46.3 & 45.5 & 47.1 & 43.7 & 42.2 & 45.2 & 46.9 & 46.1 & 47.8 \\
\hline Smoking & No & 50.5 & 50.3 & 50.8 & 45.6 & 44.7 & 46.6 & 51.2 & 51.0 & 51.5 \\
\hline \multirow{3}{*}{ Health Status } & Excellent/Very good & 49.1 & 48.7 & 49.4 & 46.8 & 45.7 & 47.9 & 49.4 & 49.1 & 49.8 \\
\hline & Good & 53.5 & 53.3 & 53.8 & 53.1 & 52.3 & 54.0 & 53.5 & 53.3 & 53.8 \\
\hline & Fair/poor & 40.4 & 39.8 & 41.1 & 36.6 & 35.6 & 37.6 & 42.0 & 41.3 & 42.7 \\
\hline \multirow{2}{*}{ Diabetes } & Yes & 41.0 & 40.1 & 41.9 & 35.9 & 34.2 & 37.6 & 42.7 & 41.7 & 43.6 \\
\hline & No & 51.1 & 50.8 & 51.3 & 47.5 & 46.9 & 48.2 & 51.6 & 51.3 & 51.8 \\
\hline \multirow{2}{*}{ Hypertension } & Yes & 44.3 & 43.6 & 44.9 & 40.0 & 38.8 & 41.2 & 45.4 & 44.7 & 46.2 \\
\hline & No & 52.0 & 51.7 & 52.2 & 49.1 & 48.4 & 49.9 & 52.3 & 52.0 & 52.5 \\
\hline \multirow{2}{*}{$\begin{array}{l}\text { High } \\
\text { cholesterol }\end{array}$} & Yes & 45.4 & 44.8 & 46.0 & 40.8 & 39.6 & 42.0 & 46.6 & 46.0 & 47.1 \\
\hline & No & 51.6 & 51.4 & 51.9 & 48.2 & 47.4 & 49.1 & 52.0 & 51.8 & 52.3 \\
\hline \multirow{2}{*}{ Heart disease } & Yes & 41.1 & 40.2 & 42.1 & 37.0 & 35.2 & 38.8 & 42.8 & 41.8 & 43.8 \\
\hline & No & 50.9 & 50.7 & 51.2 & 47.4 & 46.7 & 48.2 & 51.4 & 51.2 & 51.7 \\
\hline Cancer & No & 50.3 & 50.1 & 50.6 & 46.0 & 45.2 & 46.7 & 51.0 & 50.7 & 51.2 \\
\hline \multirow{4}{*}{ Year } & 2013 & 50.0 & 49.6 & 50.4 & 44.8 & 43.7 & 45.9 & 50.7 & 50.3 & 51.2 \\
\hline & 2014 & 50.2 & 49.8 & 50.7 & 45.3 & 44.0 & 46.6 & 50.8 & 50.3 & 51.2 \\
\hline & 2015 & 49.9 & 49.5 & 50.3 & 44.7 & 43.2 & 46.1 & 50.6 & 50.2 & 51.0 \\
\hline & 2016 & 50.0 & 49.6 & 50.5 & 46.5 & 45.2 & 47.9 & 50.9 & 50.5 & 51.3 \\
\hline
\end{tabular}

Note. Only patients with SF-12 scores were included. PCS and MCS are not available for 2017 MEPS data. 
Table 3. Mental Component Summary (MCS)

\begin{tabular}{|c|c|c|c|c|c|c|c|c|c|c|}
\hline \multirow{2}{*}{ Category } & & \multicolumn{3}{|c|}{ Overall } & \multicolumn{3}{|c|}{ Mental Disorder } & \multicolumn{3}{|c|}{ Non-Mental Disorder } \\
\hline & & Mean & $\begin{array}{c}\text { Lower } \\
95 \% \text { CI }\end{array}$ & $\begin{array}{c}\text { Upper } \\
95 \% \text { CI }\end{array}$ & Mean & $\begin{array}{c}\text { Lower } \\
95 \% \text { CI }\end{array}$ & $\begin{array}{c}\text { Upper } \\
95 \% \text { CI }\end{array}$ & Mean & $\begin{array}{c}\text { Lower } \\
95 \% \mathrm{CI}\end{array}$ & $\begin{array}{c}\text { Upper } \\
95 \% \text { CI }\end{array}$ \\
\hline Overall & & 51.3 & 51.0 & 51.5 & 42.6 & 42.0 & 43.3 & 52.6 & 52.3 & 52.8 \\
\hline \multirow{3}{*}{ Age } & $18-44$ & 51.9 & 51.6 & 52.3 & 43.0 & 42.1 & 43.9 & 53.0 & 52.7 & 53.3 \\
\hline & $45-64$ & 50.7 & 50.3 & 51.1 & 42.3 & 41.3 & 43.3 & 52.2 & 51.8 & 52.6 \\
\hline & $\geq 65$ & 49.0 & 48.1 & 49.8 & 42.1 & 40.3 & 43.9 & 50.6 & 49.7 & 51.4 \\
\hline \multirow{2}{*}{ Race } & White & 51.3 & 51.1 & 51.6 & 42.8 & 42.1 & 43.4 & 52.6 & 52.3 & 52.8 \\
\hline & Others & 49.7 & 48.6 & 50.7 & 40.5 & 38.3 & 42.8 & 52.1 & 51.2 & 52.9 \\
\hline \multirow{4}{*}{ Region } & Northeast & 50.2 & 49.4 & 50.9 & 41.1 & 39.8 & 42.4 & 52.0 & 51.2 & 52.7 \\
\hline & Midwest & 51.3 & 50.3 & 52.2 & 43.3 & 41.1 & 45.6 & 53.0 & 52.3 & 53.7 \\
\hline & South & 51.7 & 51.3 & 52.1 & 42.7 & 41.5 & 43.8 & 52.7 & 52.4 & 53.1 \\
\hline & West & 51.3 & 50.8 & 51.7 & 43.0 & 42.0 & 44.0 & 52.5 & 52.1 & 52.9 \\
\hline \multirow[b]{2}{*}{ Insurance Type } & Any private & 52.5 & 52.2 & 52.8 & 45.5 & 44.5 & 46.4 & 53.5 & 53.3 & 53.8 \\
\hline & Public only & 48.4 & 47.9 & 48.9 & 42.4 & 40.9 & 43.8 & 50.9 & 50.5 & 51.3 \\
\hline Marital Status & Unmarried & 50.8 & 50.4 & 51.1 & 41.7 & 40.8 & 42.5 & 52.4 & 52.1 & 52.7 \\
\hline \multirow{3}{*}{$\begin{array}{l}\text { Education } \\
\text { Attainment }\end{array}$} & Less than high school & 49.8 & 49.4 & 50.3 & 40.5 & 39.5 & 41.5 & 51.1 & 50.7 & 51.6 \\
\hline & High school & 52.0 & 51.6 & 52.3 & 43.8 & 42.7 & 44.9 & 53.0 & 52.7 & 53.4 \\
\hline & College or higher & 52.0 & 51.6 & 52.4 & 43.4 & 42.5 & 44.4 & 53.5 & 53.2 & 53.8 \\
\hline \multirow{3}{*}{ Family Income } & Negative/poor/low & 52.1 & 51.7 & 52.5 & 44.5 & 43.4 & 45.5 & 53.1 & 52.7 & 53.5 \\
\hline & Middle & 53.0 & 52.6 & 53.4 & 46.1 & 45.0 & 47.2 & 53.9 & 53.5 & 54.4 \\
\hline & High & 49.8 & 49.4 & 50.1 & 40.1 & 39.3 & 40.9 & 51.4 & 51.1 & 51.8 \\
\hline \multirow{3}{*}{$\begin{array}{l}\text { Current } \\
\text { Smoking }\end{array}$} & Yes & 46.4 & 45.5 & 47.2 & 38.4 & 37.0 & 39.9 & 48.3 & 47.4 & 49.2 \\
\hline & No & 51.9 & 51.6 & 52.2 & 43.5 & 42.8 & 44.2 & 53.1 & 52.8 & 53.3 \\
\hline & Excellent/Very good & 50.4 & 50.1 & 50.8 & 46.2 & 45.1 & 47.3 & 51.5 & 51.1 & 51.9 \\
\hline \multirow[t]{2}{*}{ Health Status } & Good & 53.7 & 53.4 & 54.0 & 43.6 & 42.7 & 44.5 & 54.3 & 54.0 & 54.6 \\
\hline & Fair/poor & 44.9 & 44.4 & 45.5 & 38.4 & 37.3 & 39.4 & 47.6 & 47.0 & 48.1 \\
\hline \multirow{2}{*}{ Diabetes } & Yes & 47.8 & 47.0 & 48.6 & 40.6 & 39.0 & 42.2 & 50.1 & 49.4 & 50.9 \\
\hline & No & 51.7 & 51.4 & 51.9 & 43.1 & 42.4 & 43.8 & 52.8 & 52.5 & 53.1 \\
\hline Hypertension & Yes & 48.7 & 48.2 & 49.1 & 41.3 & 40.4 & 42.3 & 50.8 & 50.3 & 51.2 \\
\hline $\begin{array}{l}\text { High } \\
\text { cholesterol }\end{array}$ & No & 52.0 & 51.7 & 52.3 & 43.0 & 42.2 & 43.7 & 53.1 & 52.8 & 53.3 \\
\hline \multirow{2}{*}{ Heart disease } & Yes & 47.0 & 46.3 & 47.8 & 40.0 & 38.5 & 41.6 & 49.9 & 49.2 & 50.6 \\
\hline & No & 51.7 & 51.4 & 52.0 & 43.3 & 42.6 & 44.0 & 52.8 & 52.5 & 53.0 \\
\hline \multirow{2}{*}{ Arthritis } & Yes & 47.9 & 47.2 & 48.5 & 40.3 & 39.2 & 41.4 & 50.7 & 50.0 & 51.3 \\
\hline & No & 51.9 & 51.6 & 52.2 & 43.7 & 42.9 & 44.5 & 52.8 & 52.6 & 53.1 \\
\hline \multirow{2}{*}{ Cancer } & Yes & 48.6 & 47.5 & 49.7 & 41.3 & 38.3 & 44.3 & 50.9 & 49.8 & 52.0 \\
\hline & No & 51.4 & 51.1 & 51.7 & 42.7 & 42.1 & 43.4 & 52.6 & 52.4 & 52.9 \\
\hline \multirow{4}{*}{ Year } & 2013 & 50.7 & 50.3 & 51.2 & 41.8 & 40.8 & 42.9 & 52.0 & 51.6 & 52.5 \\
\hline & 2014 & 51.6 & 51.2 & 52.0 & 42.3 & 41.2 & 43.3 & 52.8 & 52.4 & 53.2 \\
\hline & 2015 & 51.4 & 50.9 & 51.9 & 43.6 & 42.5 & 44.7 & 52.8 & 52.4 & 53.3 \\
\hline & 2016 & 51.3 & 50.9 & 51.7 & 42.7 & 41.5 & 43.8 & 52.5 & 52.1 & 52.9 \\
\hline
\end{tabular}

Note. Only patients with SF-12 scores were included. PCS and MCS are not available for 2017 MEPS data.

\section{Discussion}

This study's objective was to identify relevant factors that affect Hispanic patients with mental health problems' HRQoL scores to distinguish disparities that must be addressed. HRQoL scores, which include physical, mental, and perceived health and social well-being, are essential factors determining treatment outcomes. ${ }^{[8,9]}$ They are affected by various factors, including age, race, education, socio-geographic, and socioeconomic status. ${ }^{[10,12-14]}$ These influencing factors are enormously consequential for Hispanic patients with mental disorders when analyzing HRQoL scores and treatment outcomes because of the large socioeconomic and health disparities already felt by this demographic. ${ }^{[16,17]}$
Overall, many Hispanic patients are uninsured, at a rate of $26.1 \%$, which may impede their ability to seek treatment. A higher percentage of patients with mental health problems are over 65 years old $(15.5 \%)$ than those without mental health problems $(9.6 \%)$. The mental disorder group has higher education rates than the overall and non-mental disorder group, with $46.5 \%$ of patients having attended college or higher compared to $39.2 \%$ and $38.2 \%$, respectively. Most respondents reported having a low family income, but this percentage was significantly higher in Hispanic patients with mental disorders at a rate of $48.8 \%, 6.7 \%$ higher than those without a mental disorder. 
Table 4. Demographic characteristics associated with SF-12 among Mental disorder vs. non-Mental disorder

\begin{tabular}{|c|c|c|c|c|c|c|c|c|c|c|}
\hline \multirow[b]{2}{*}{ Category } & \multicolumn{5}{|c|}{ Physical Component Summary (PCS) } & \multicolumn{5}{|c|}{ Mental Component Summary (MCS) } \\
\hline & Estimate & $t$ & $\begin{array}{c}\text { Lower } \\
95 \% \text { CI }\end{array}$ & $\begin{array}{c}\text { Upper } \\
95 \% \text { CI }\end{array}$ & $p$ & Estimate & $\mathbf{t}$ & $\begin{array}{c}\text { Lower } \\
95 \% \text { CI }\end{array}$ & $\begin{array}{c}\text { Upper } \\
95 \% \text { CI }\end{array}$ & $p$ \\
\hline \multicolumn{11}{|l|}{ Mental disorder } \\
\hline Mental disorder & Ref. & & & & & Ref. & & & & \\
\hline Non-Mental disorder & 1.10 & 3.77 & 0.53 & 1.67 & .000 & 7.83 & 26.70 & 7.25 & 8.40 & $<.0001$ \\
\hline \multicolumn{11}{|l|}{ Age } \\
\hline $18-44$ & Ref. & & & & & Ref. & & & & \\
\hline $45-64$ & -1.90 & -8.00 & -2.37 & -1.44 & $<.0001$ & 0.44 & 1.93 & -0.01 & 0.88 & .054 \\
\hline$\geq 65$ & -4.16 & -10.83 & -4.92 & -3.41 & $<.0001$ & 0.71 & 1.62 & -0.15 & 1.58 & .105 \\
\hline \multicolumn{11}{|l|}{ Race/ethnicity } \\
\hline White, Hispanic & Ref. & & & & & Ref. & & & & \\
\hline Black, Hispanic & 0.85 & 1.77 & -0.09 & 1.79 & .077 & 0.14 & 0.28 & -0.83 & 1.10 & .780 \\
\hline Others, Hispanic & 0.68 & 1.71 & -0.10 & 1.46 & .089 & -0.65 & -1.43 & -1.55 & 0.25 & .154 \\
\hline \multicolumn{11}{|l|}{ Region } \\
\hline Northeast & Ref. & & & & & Ref. & & & & \\
\hline Midwest & 0.13 & 0.31 & -0.72 & 0.98 & .758 & 0.79 & 1.64 & -0.16 & 1.74 & .101 \\
\hline South & -0.15 & -0.47 & -0.76 & 0.46 & .637 & 0.38 & 1.07 & -0.32 & 1.07 & .287 \\
\hline West & 0.02 & 0.06 & -0.58 & 0.62 & .953 & 0.35 & 0.94 & -0.37 & 1.07 & .346 \\
\hline \multicolumn{11}{|l|}{ Insurance Type } \\
\hline Any private & Ref. & & & & & Ref. & & & & \\
\hline Public only & -1.91 & -7.41 & -2.42 & -1.40 & $<.0001$ & -1.14 & -4.23 & -1.67 & -0.61 & $<.0001$ \\
\hline Uninsured & -0.14 & -0.62 & -0.60 & 0.31 & .533 & -0.51 & -2.00 & -1.01 & -0.01 & .047 \\
\hline \multicolumn{11}{|l|}{ Marital Status } \\
\hline Married & Ref. & & & & & Ref. & & & & \\
\hline Unmarried & 0.31 & 1.74 & -0.04 & 0.65 & .082 & -0.41 & -2.15 & -0.78 & -0.04 & .032 \\
\hline \multicolumn{11}{|l|}{ Education Attainment } \\
\hline Less than high school & Ref. & & & & & Ref. & & & & \\
\hline High school & 0.41 & 1.64 & -0.08 & 0.90 & .101 & 1.27 & 5.14 & 0.79 & 1.76 & $<.0001$ \\
\hline College or higher & 0.56 & 2.26 & 0.07 & 1.04 & .024 & 0.72 & 3.08 & 0.26 & 1.18 & .002 \\
\hline \multicolumn{11}{|l|}{ Family Income a } \\
\hline Negative/poor/low & Ref. & & & & & Ref. & & & & \\
\hline Middle & 0.58 & 3.11 & 0.21 & 0.94 & .002 & 0.96 & 4.15 & 0.50 & 1.41 & $<.0001$ \\
\hline High & 0.97 & 3.80 & 0.47 & 1.47 & $<.000$ & 1.02 & 3.48 & 0.44 & 1.60 & .001 \\
\hline \multicolumn{11}{|l|}{ Current Smoking } \\
\hline Yes & Ref. & & & & & Ref. & & & & \\
\hline No & 3.22 & 8.35 & 2.46 & 3.98 & $<.0001$ & 4.30 & 9.94 & 3.45 & 5.15 & $<.0001$ \\
\hline Health Status & & & & & & & & & & \\
\hline Excellent/Very good & Ref. & & & & & Ref. & & & & \\
\hline Good & -2.63 & -15.23 & -2.97 & -2.29 & $<.0001$ & -2.25 & -11.10 & -2.65 & -1.85 & $<.0001$ \\
\hline Fair/poor & -7.71 & -25.02 & -8.31 & -7.10 & $<.0001$ & -5.68 & -20.71 & -6.22 & -5.14 & $<.0001$ \\
\hline Diabetes & & & & & & & & & & \\
\hline Yes & Ref. & & & & & Ref. & & & & \\
\hline No & 2.09 & 6.78 & 1.48 & 2.69 & $<.0001$ & -0.08 & -0.24 & -0.77 & 0.60 & .810 \\
\hline Hypertension & & & & & & & & & & \\
\hline Yes & Ref. & & & & & Ref. & & & & \\
\hline No & 1.15 & 4.82 & 0.68 & 1.62 & $<.0001$ & 0.48 & 2.03 & 0.02 & 0.95 & .043 \\
\hline High cholesterol & & & & & & & & & & \\
\hline Yes & Ref. & & & & & Ref. & & & & \\
\hline No & 0.28 & 1.27 & -0.15 & 0.71 & .204 & 0.62 & 2.32 & 0.09 & 1.15 & .021 \\
\hline Heart disease & & & & & & & & & & \\
\hline Yes & Ref. & & & & & Ref. & & & & \\
\hline No & 2.89 & 8.87 & 2.25 & 3.53 & $<.0001$ & 1.02 & 2.82 & 0.31 & 1.72 & .005 \\
\hline Arthritis & & & & & & & & & & \\
\hline Yes & Ref. & & & & & Ref. & & & & \\
\hline No & 4.87 & 16.49 & 4.29 & 5.45 & $<.0001$ & 0.34 & 1.10 & -0.27 & 0.95 & .272 \\
\hline Cancer & & & & & & & & & & \\
\hline Yes & Ref. & & & & & Ref. & & & & \\
\hline No & 0.85 & 1.70 & -0.13 & 1.83 & .091 & 0.52 & 0.99 & -0.52 & 1.56 & .325 \\
\hline Year & & & & & & & & & & \\
\hline 2013 & Ref. & & & & & Ref. & & & & \\
\hline 2014 & 0.10 & 0.44 & -0.35 & 0.55 & .657 & 0.49 & 1.88 & -0.02 & 1.00 & .061 \\
\hline 2015 & -0.03 & -0.11 & -0.59 & 0.53 & .909 & 0.53 & 1.80 & -0.05 & 1.11 & .073 \\
\hline 2016 & 0.09 & 0.38 & -0.40 & 0.58 & .706 & 0.44 & 1.65 & -0.08 & 0.96 & .099 \\
\hline
\end{tabular}


Comparing SF-12 PCS and MCS scores in Hispanic patients with mental health disorders, a clear and significant trend appears. The difference in MCS scores between the two groups was twice as high as in the PCS scores. Those with mental health disorders had markedly lower PCS and MCS scores for health status, reflecting how their condition may affect their general well-being. Similarly, patients without comorbidities had much higher PCS and slightly higher MCS scores than those with comorbidities across almost all sampled conditions. This trend is reflected across various health factors, including smoking, with non-smoking patients scoring higher in PCS and MCS. These findings are significant because of the implication that health status is affected by mental and physical well-being.

In the United States, health insurance is a vital necessity to receiving care. Past studies have described how insurance status leads to increased healthcare utilization. ${ }^{[23,24]}$ Most of the overall group was either privately $(49.3 \%)$ or publicly (24.6\%) insured, but many Hispanic patients remain uninsured $(26.1 \%)$. Many patients were privately insured, with $46.8 \%$ of patients with mental health problems and $49.6 \%$ of patients without mental disorders having private insurance. The percent of mental health patients with public insurance $(40.4 \%)$ was almost double that of those without mental health problems using public insurance $(22.4 \%)$. Patients with mental disorders were more likely to be insured than those without mental disorders. Only $12.8 \%$ of those with mental health problems were uninsured, $10 \%$ less than those without mental health problems.

Focusing on health status and comorbidities, more than half of patients without mental disorders reported having excellent health; only a more minor third of patients with mental disorders could make the same claim. Most patients with mental disorders, about $34 \%$, felt that their health status was low, compared to $12.7 \%$ of patients without mental disorders. Overall, patients reporting fair to low health status reported much lower PCS and MCS scores compared to those who reported having good or excellent/very good health status. Also, PCS scores and MCS scores were lower in patients with mental disorders reporting low health status than patients without mental disorders reporting low health status. Based on the above findings, low perceived health status and HRQoL scores in patients are affected more by the presence of a mental disorder. Across multiple comorbidities, a greater rate of patients with mental disorders tended to suffer from various conditions than those without mental disorders. The rate of hypertension, diabetes, high cholesterol, heart disease, arthritis, and cancer was about twice as high in patients with mental disorders than those without mental disorders. These findings are consistent with previous findings that exhibited how patients with mental health problems have higher comorbidity rates. ${ }^{[25]}$ The presence of comorbidities resulted in lower PCS and MCS scores with a higher disparity in PCS. When considering the demographic's high rate of low income and lack of insurance, having multiple comorbidities, including mental disorders, can significantly affect HRQoL scores.

This study has several limitations. Its cross-section design cannot explain causation; thus, the results' interpretation is restrained to the association between comorbid anxiety and mood disorders to HRQoL. Also, recall bias is possible because medical conditions in MEPS are self- or proxyreported and not verified by chart review. Because the general population was involved, a generic tool was used for a controlled comparison. Generic measures are capable of a broad range of HRQoL dimensions with a single instrument but are not as responsive as specific tools focused on a particular disease. Lastly, CCI may not have captured more common comorbidities that might influence the results of this study. However, this study remains the first to present unique data on the HRQoL of the Hispanic population with mental disorders in the United States despite these limitations.

\section{Conclusions}

In conclusion, significant disparities exist in HRQoL between patients with mental health disorders and patients without mental health disorders in the Hispanic population. Through SF-12 PCS and MCS surveys, this study found that patients with mental health disorders experience lower HRQoL than those without mental health disorders. Lower family income, lower perceived health status, and comorbidities are factors that significantly lower the HRQoL of patients. We hope this study highlights the impact of mental health disorders in HRQoL in the Hispanic population and leads to further research to identify ways to improve HRQoL in this patient population.

\section{CONFLICTS OF INTEREST DisClOSURE}

The authors declare they have no conflicts of interest. 


\section{REFERENCES}

[1] What Is Mental Illness? Psychiatry.org. Available from: https://www.psychiatry.org/patients-families/what -is-mental-illness

[2] Mental Disorders. U.S. National Library of Medicine. MedlinePlus.gov. Available from: https://medlineplus.gov/mental disorders.html

[3] Mental Health By the Numbers. National Alliance of Mental Health. Nami.org. Available from: https://www.nami.org/mhstats

[4] Latinx/Hispanic Communities and Mental Health. Mental health America. Mhanational.org. Available from: https://www.mhanational .org/issues/latinxhispa nic-communities-and-mental-health

[5] Cook EL, Harman JS. A comparison of health-related quality of life for individuals with mental health disorders and common chronic medical conditions. Public Health Rep. 2008; 123(1): 45-51. PMid: 18348479. https ://doi.org/10.1177/003335490812300107

[6] QuickFacts United States. United States Census Bureau. Census.gov. Available from: https://www.census.gov/quickfacts/fact /table/US/RHI725219

[7] Latinx/Hispanic. National Alliance of Mental Health. Nami.org. Available from: https://www.nami.org/Your-Journey/Ide ntity-and-Cultural-Dimensions/Latinx-Hispanic

[8] Sitlinger A, Zafar SY. Health-Related Quality of Life: The Impact on Morbidity and Mortality. Surg Oncol Clin N Am. 2018; 27(4): 675-684. PMid: 30213412. https://doi.org/10.1016/j.soc. 2018.05.008

[9] Sosnowski R, Kulpa M, Ziętalewicz U, et al. Basic issues concerning health-related quality of life. Cent European J Urol. 2017; 70(2): 206-211. https://doi.org/10.5173/ceju.2017.923

[10] Kagawa-Singer M, Padilla G, Ashing-Giwa K. Health-Related Quality of Life and Culture. Seminars in Oncology Nursing. 2010; 26(1): 59-67. PMid: 20152579. https://doi.org/10.1016/j.soncn. 2009.11.008

[11] Etxeberria I, Urdaneta E, Galdona N. Factors associated with healthrelated quality of life (HRQoL): differential patterns depending on age. Qual Life Res. 2019; 28(8): 2221-2231. PMid: 31065936. https : //doi.org/10.1007/s11136-019-02182-0

[12] Kolotkin RL, Andersen JR. A systematic review of reviews: exploring the relationship between obesity, weight loss and health-related quality of life. Clin Obesity. 2017; 7: 273-289. PMid: 28695722. https://doi.org/10.1111/cob.12203

[13] Laxy M, Teuner C, Holle R, et al. The association between BMI and health-related quality of life in the US population: sex, age and ethnicity matters. International Journal of Obesity. 2018; 42: 318-326. PMid: 28993709. https ://doi.org/10.1038/ijo.2017.252
[14] Wallander JL, Fradkin C, Elliott MN, et al. Racial/ethnic disparities in health-related quality of life and health status across pre-, early-, and mid-adolescence: a prospective cohort study. Quality of Life Research. 2019; 28: 1761-1771. PMid: 30927145. https ://doi.org/10.1007/s11136-019-02157-1

[15] Chowdhury PP, Balluz L, Strine TW. Health-related quality of life among minority populations in the United States, BRFSS 2001-2002. Ethn Dis. 2008; 18(4): 483-487.

[16] Garcini LM, Renzaho A, Molina M, et al. Health-related quality of life among Mexican-origin Latinos: the role of immigration legal status. Ethnicity \& Health. 2018; 23(5): 566-581. PMid: 28158950. https://doi.org/10.1080/13557858.2017.1283392

[17] Santee EJ, King KA, Vidourek RA, et al. Health Care Access and Health Behavior Quality of Life among Hispanic/Latino-American Cancer Survivors. Applied Research Quality Life. 2020; 15: 637-650. https ://doi.org/10.1007/s11482-018-9677-1

[18] AHRQ. MEPS summary tables. 2019 [Accessed September 29, 2019] Available from: https://meps.ahrq.gov/mepstrends/hc_co nd/

[19] Ware J. Jr., Kosinski M, Keller SD. A 12-Item Short-Form Health Survey: construction of scales and preliminary tests of reliability and validity. Med Care. 1996; 34(3): 220-233. PMid: 8628042 https : //doi.org/10.1097/00005650-199603000-00003

[20] MEPS HC-192 2016 Full Year Consolidated Data File. Agency for Healthcare Research and Quality (AHRQ), August 2018.

[21] Baernholdt M, Hinton I, Yan G, et al. Factors associated with quality of life in older adults in the United States. Qual Life Res. 2012; 21(3): 527-534. PMid: 21706127. https ://doi.org/10.1007/s11136 $-011-9954-z$

[22] Using Statistical Software Packages to Produce Estimates from MEPS Data File. Agency for Healthcare Research and Quality (AHRQ). 2018.

[23] Steele LS, Dewa CS, Lin E, et al. Education level, income level and mental health services use in Canada: associations and policy implications. Healthc Policy. 2007; 3(1): 96-106. PMid: 19305758 https://doi.org/10.12927/hcpol.2007.19177

[24] National Academies of Sciences, Engineering, and Medicine; Health and Medicine Division; Board on Health Care Services; Committee on Health Care Utilization and Adults with Disabilities. Health-Care Utilization as a Proxy in Disability Determination. Washington (DC): National Academies Press (US); March 1, 2018.

[25] Momen NC, Plana-Ripoll O, Agerbo E, et al. Association between Mental Disorders and Subsequent Medical Conditions. N Engl J Med. 2020; 382(18): 1721-1731. PMid: 32348643. https : //doi.org/10.1056/NEJMoa1915784 